\title{
The use of hyperbaric oxygen therapy in actinomycotic osteomyelitis associa- ted florid cemento osseous dysplasia: report of a familial case.
}

\author{
Sushma Mehkri' ${ }^{1}$ K.S. Nagesh ${ }^{2}$, Asha R Iyengar ${ }^{3}$, S. Girish Rao ${ }^{4}$ \\ ${ }^{1}$ M.D.S., Lecturer, Department of Oral Medicine and Radiology, DAPM R.V. Dental College and Hospital, Bangalore \\ ${ }^{2}$ M.D.S., HOD and Principal, Department of Oral Medicine and Radiology, DAPM R.V. Dental College and Hospital, Bangalore \\ ${ }^{3}$ M.D.S., Professor, Department of Oral Medicine and Radiology, DAPM R.V. Dental College and Hospital, Bangalore \\ ${ }^{4}$ M.D.S., FDSRCS (ENG), FFDRCSI (IRE), Professor and HOD, Department of Oral and Maxillofacial Sur-gery, DAPM R.V. \\ Dental College and Hospital, Bangalore.
}

Correspondence:

Dr. Sushma Mehkri

DAPM R.V. Dental College and Hospital

CA-37, 24th main, 1st phase J.P. Nagar,

Bangalore -560078

India

e-mail address: sushmamehkri@gmail.com.

Received: $16 / 03 / 2010$

Accepted: 01/05/2010
Mehkri S, Nagesh KS, Iyengar AR, Rao SG. The use of hyperbaric oxygen therapy in actinomycotic osteomyelitis associated florid cemento osseous dysplasia: report of a familial case. J Clin Exp Dent. 2010;2 (3):e121-6.

http://www.medicinaoral.com/odo/volumenes/v2i3/jcedv2i3p121.pdf (C) Medicina Oral S. L. C.I.F. B 96689336 - eISSN: $1989-5488$

eMail: jced@jced.com

\begin{abstract}
Florid Cemento-Osseous Dysplasia (FCOD) is a well recognized fibro-osseous disease of the jaws commonly seen in the middle aged African women, although it may occur in the Caucasians and Asians. There is a reported incidence of less than $2 \%$ in the Indian population.

The clinical and the radiographic features of FCOD may overlap with features of chronic diffuse scle-rosing osteomyelitis, which makes diagnosis of the disease difficult in symptomatic cases. Moreover the jaws may be susceptible to osteomyelitis, which may mask the underlying FCOD in some cases.

The case reported here is that of a 56 year old Indian female patient with long standing FCOD invol-ving the maxilla and the mandible, who was asymptomatic till all her teeth were extracted for fabrica-tion of complete dentures. The patient experienced symptoms of pain, swelling and purulent discharge soon after the use of dentures and was treated by surgical removal of the affected bone. The same symptoms recurred in other areas of the jaw over a period of time and these areas were treated surgica-lly. The patient subsequently presented with pain in the mandibular left posterior region of ten days duration, and was diagnosed as FCOD with superimposed Actinomycotic Osteomyelitis based on his-topathology and Gram's stain, and was treated by surgical removal of the affected bone followed by hyperbaric oxygen therapy (HBO). The radiograph of the surgical site showed good amount of bone regeneration within six months of HBO therapy without recurrence of the FCOD in the same area till date.
\end{abstract}

Key words: Hyperbaric oxygenation, florid cemento osseous dysplasia, osteomyelitis, panoramic radiography. 


\section{Introduction}

Bone dysplasias constitute a group of conditions in which normal bone is replaced with fibrous tissue containing abnormal bone or cementum (1). These are commonly called as 'Fibro osseous lesions' and include fibrous dysplasia, ossifying fibroma, periapical cemental dysplasia (PCD), florid cemento-osseous dysplasia (FCOD), focal cemento-osseous dysplasia $(1,2)$. All the above lesions present as radiolucent to radioopaque areas in different parts the jaws. PCD is associated with the apices of man-dibular anterior teeth, whereas FCOD is multifocal sometimes involving all the four quadrants. Both the lesions are common in middle aged black women with the periapex of the tooth being the epicentre of the lesion and with similar radiographic and histologic appearance. The lesions of focal cemento-osseous dysplasia are similar to the lesions of PCD or FCOD but involve only focal areas of mandibu-lar posterior region (2).

FCOD is a reactive fibro-osseous lesion of the periodontal ligament origin (3) which is commonly seen in the middle aged African women, although it may occur in the Caucasians and Asians. There is a reported incidence of less than $2 \%$ in the Indian population (4).

The disease process may be completely asymptomatic, and in such cases the lesions are detected when the radiographs are taken for some other purposes (3). Symptoms such as dull pain or drainage are always associated with exposure of the sclerotic calcified masses in the oral cavity (3). This may occur as a result of progressive alveolar atrophy under a denture or after extraction of teeth in the affected area (5). Radiographically FCOD appears as multiple sclerotic masses, located in two or more qua- drants in the tooth bearing regions often confined to the alveolar bone (5). Biopsy probably is not indi-cated for asymptomatic lesions discovered on routine radiographs (1); however the patients should be observed radiographically on a periodic basis.

This paper describes the case of a long standing symptomatic florid cemento osseous dysplasia in a 57 year old Indian female patient, who subsequently presented with superadded actinomycotic osteomye-litis and was successfully managed by surgical excision of the affected bone followed by HBO.

\section{Report of a Case}

A 56 year old Indian female patient presented to the Department of Oral and Maxillofacial Radiology 3 years back with the complaint of pain in the mandibular left posterior region of ten days duration. The pain was of dull aching type, localized and was constantly present throughout the day. There were no other associated symptoms present. Patient gives history of similar episodes of pain in other areas of the jaws since past 10-11 years, associated with purulent discharge, and sometimes swelling and ulceration of the gums. The patient had undergone full mouth extraction 12 years back and was rehabi-litated with complete removable dentures. Within a few months of the denture use she experienced pain followed by purulent discharge in the right maxillary posterior region. The panoramic radio-graphs revealed multiple sclerotic areas in the alveolar bone. The serum levels of calcium, phosp-horous and alkaline phosphatase were well within normal limits. An incisional biopsy was made in the right maxillary posterior region confir-

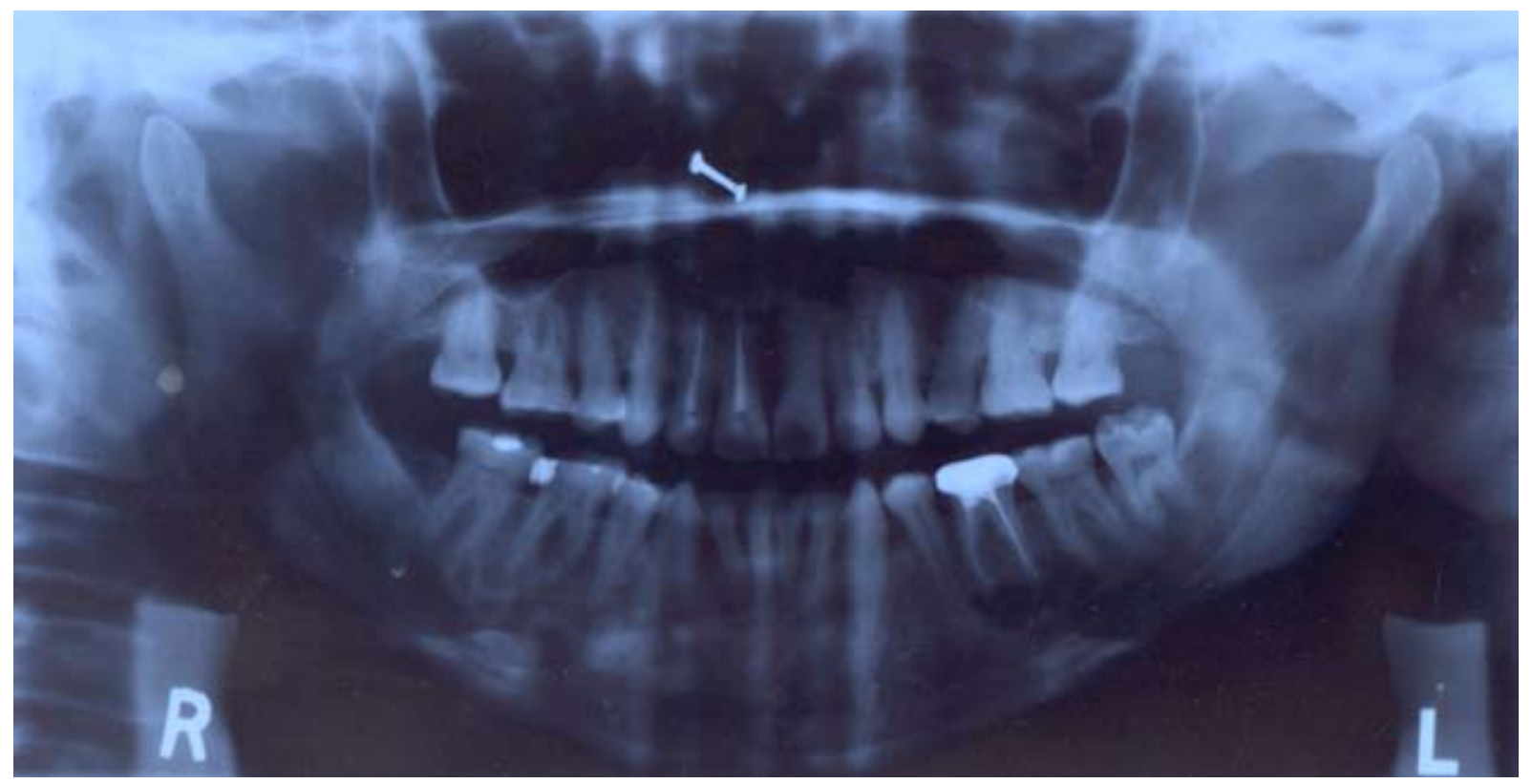

Fig.1. Panoramic radiograph of the patient's daughter revealing radiolucent areas with sclerotic borders below the apical region of 36 , 45, 46 and 47. 36 was endodontically treated a few years before the patient reported to our hospital due to the periapical lesion which are actually the lesions of FOD. 


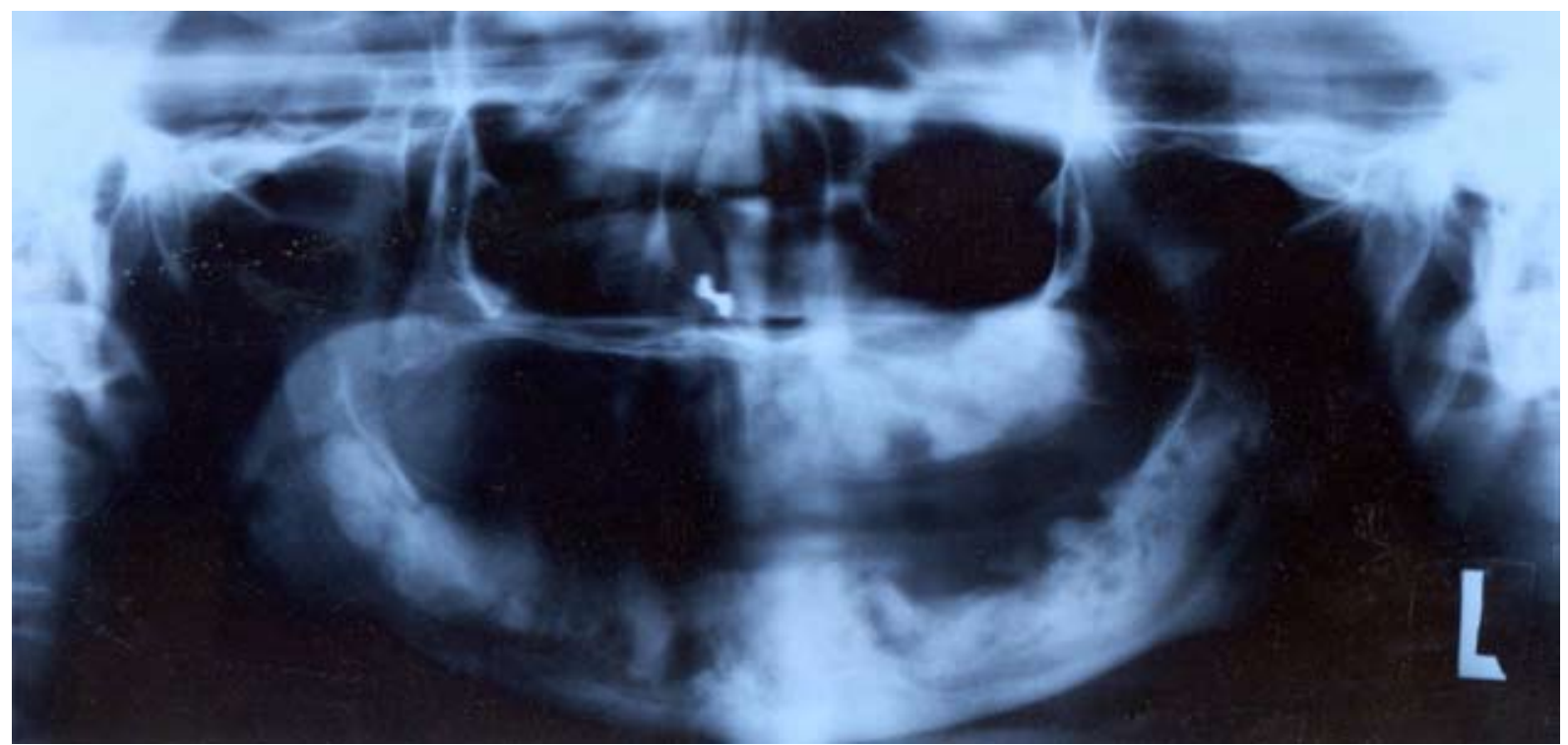

Fig.2. Panoramic radiograph revealing sclerotic areas of varying densities throughout the jaws in the region of the alveolar bone giving a "cotton wool appearance". The surface of the alveolar bone appears uneven in the mandibular left posterior region indicating the areas exposed to the oral cavity. Post-surgically treated maxillary anterior and maxillary right posterior region are also seen.

med the diagnosis of florid cemento osseous dysplasia and the affected bone was surgically excised under local anesthesia. Since then patient has experienced similar episodes of pain followed by purulent discharge in the anterior maxilla and left posterior mandible over a period of few years and has been treated by surgical removal of the affected bone. There was no relevant medical history. The patient has three children of whom the elder daughter aged 34 years had radiolucent lesions of FCOD in the radiolucent stage bilaterally in the mandible since 4 years, and has been kept under observation (Fig. 1). The patient's other two children do not have any bony involve-ment radiographically and have also been kept under observation.

On oral examination the edentulous alveolar ridges appeared to be resorbed with normal mucosal co-vering and there was no evidence of swelling, ulceration or purulent discharge. Tenderness was elici-ted in the mandibular left posterior region. The panoramic radiograph revealed sclerotic areas of diffe-rent densities throughout the jaws in the alveolar bone region giving a cotton wool appearance. The sclerotic areas were present from the anterior to left posterior region of the maxilla obscuring the floor of the maxillary sinus. The alveolar ridges were resorbed in the region of maxillary anterior and maxillary right posterior region which were the post-surgically treated areas. The sclerotic areas covered the entire mandibular alveolar bone with inferior displacement of the mandibular canal, which was more pronounced on the left side. The basal bone appeared to be normal. The

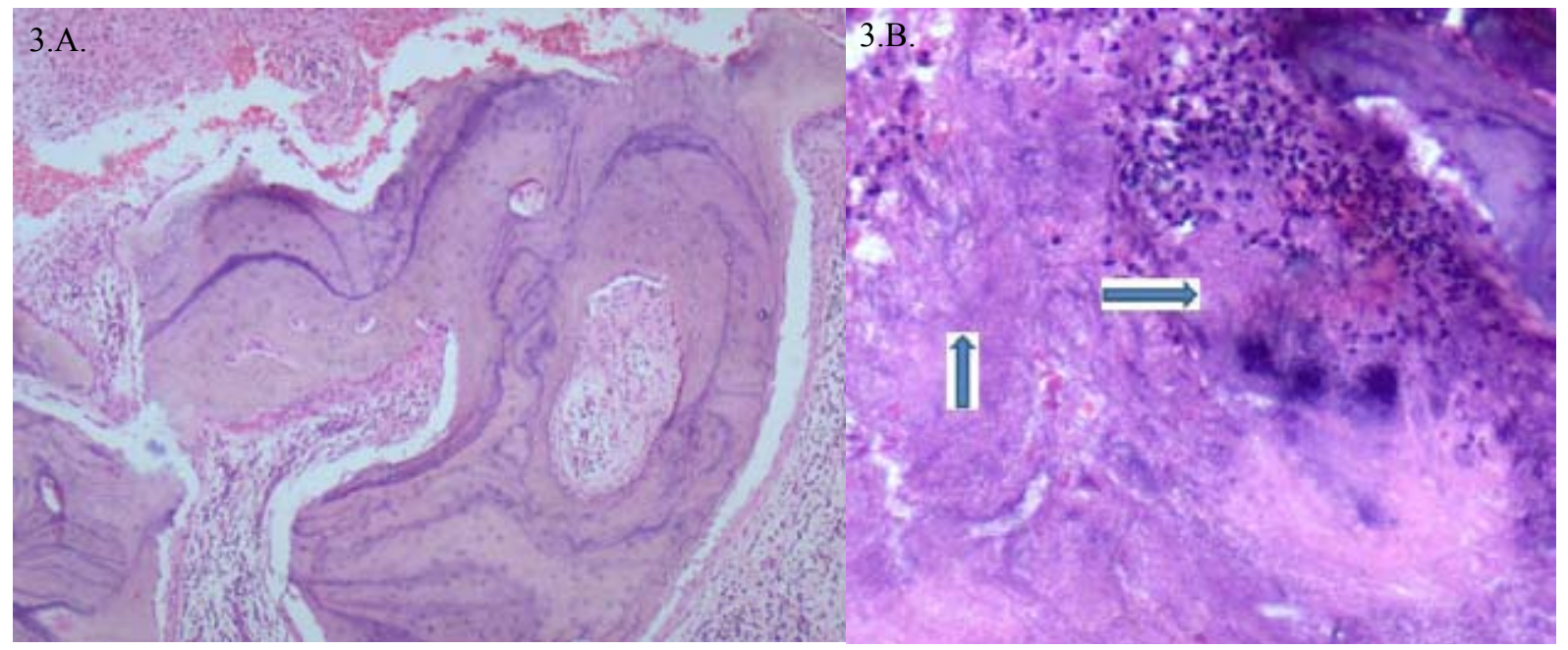

Fig 3. A. The excisional biopsy specimen showing areas of calcified masses exhibiting 'reversal lines' in a cellular fibrous stroma.

Fig 3.B. Arrows indicating actinomycotic colonies rimmed by neutrophils admist FCOD lesion. 


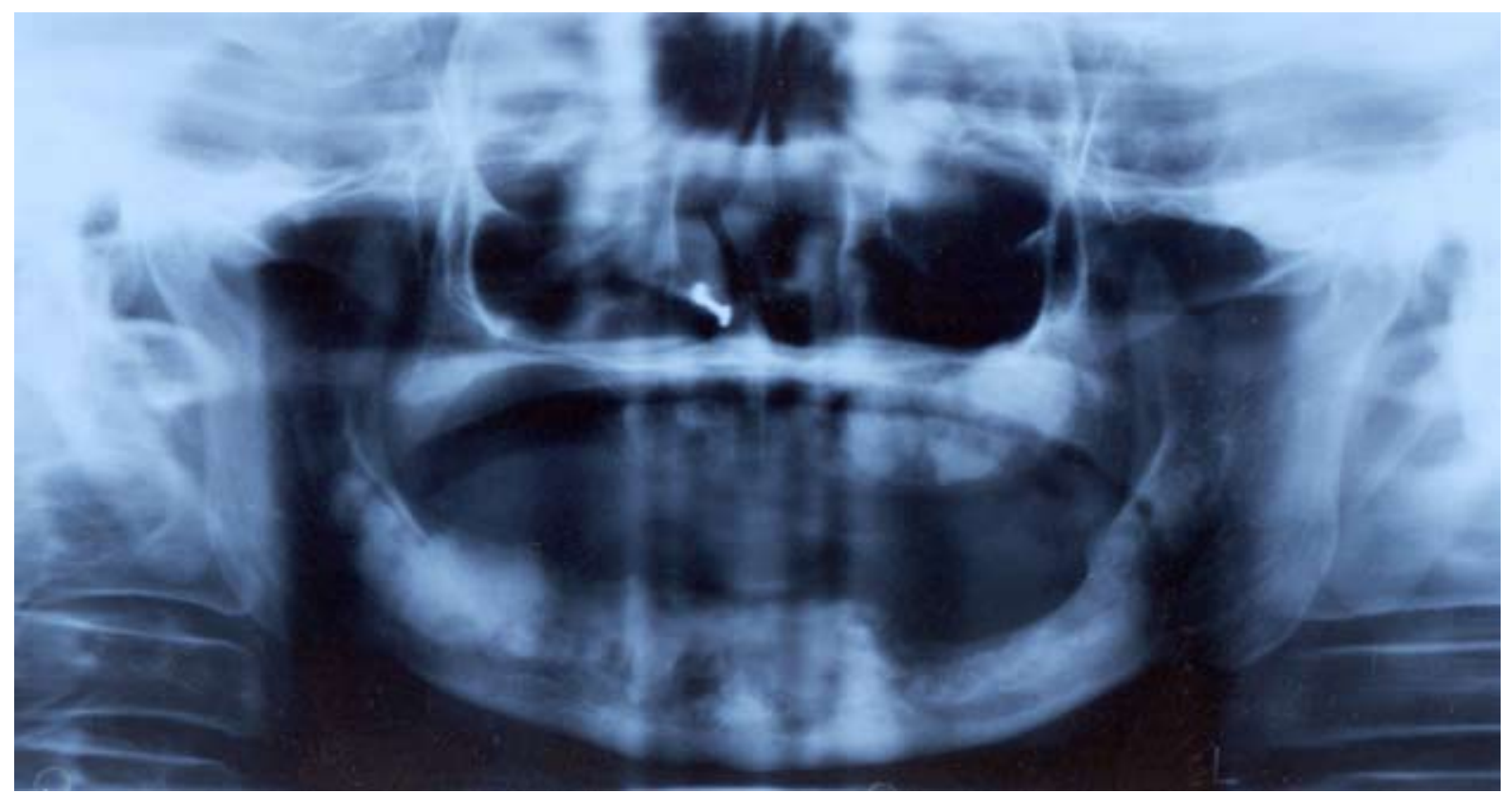

Fig.4. Panoramic radiograph 6 months after the HBO therapy revealing considerable increase in the bone density in the mandibular left posterior region

surface of the alveolar ridge in the mandibular left posterior region (molar region) was irregular with decreased bone height and with numerous radiolucent and radioopaque areas towards the surface of the ridge, indicating the cemental masses exposed to the oral cavity (Fig. 2). Surgical removal of the affected bone was done under local anesthesia in the region of mandibular left posterior region under antibiotic cover (clinda-mycin $250 \mathrm{mg}$ twice daily for 5 days). Antibiotic sensitivity and culture, and histopathological exami-nation were performed. The histopathology report revealed thick cortical bone with reactive new bone formation and dense acute inflammation, amidst which were actinomycotic colonies rimmed by neu-trophils (Fig. 3). The culture yield was a scanty growth of Staphylococcus aureus; there was no growth after 6 days in fungal culture. Zeil-Neilson stain and staining for fungi were negative and Gram's stain confirmed the diagnosis of actinomycotic osteomyelitis. The case was diagnosed as Florid Cemento Osseous Dysplasia with superimposed actinomycotic os-teomyelitis. HBO therapy was instituted using $100 \%$ oxygen at 2.5 atmospheric pressure as 10 cycles were administered (one cycle per day, five days a week for two weeks). The radiograph of the surgical site showed considerable increase in the bone density 6 months after HBO therapy (Fig. 4). The pa-tient has been kept under observation and is asymptomatic since then. The radiograph of the surgical site 3 years after the HBO therapy appeared to be normal, without recurrence of FOD lesions.

\section{Discussion}

FCOD was first described by Melrose et al. (6) in 1976 as exuberant multiquadrant masses of cemen-toma and/ or bone in both the jaws, and in some cases simple bone cavity like lesions in the affected quadrants (4). The word "Florid" was introduced to describe the widespread, extensive manifestations of the disease in the jaws (4).

The incidence of FCOD reported in the Indian population is less than 2\% (4). A review of literature from 1989 to 2009 by the authors revealed 8 cases reported from Indian population (3,4,7-9). None of the cases reported show familial tendency. The case reported here of FCOD in the mother and the daughter therefore makes it a rare presentation of FCOD in this population. In a few familial cases of FCOD that have been reported in literature, the lesions were seen in younger subjects than non-familial cases, affecting both the genders and with an autosomal dominant inheritance $(10,11)$. Some cases have been associated with bone fractures (10), abnormalities of the long bones and prepubertal pathologic fractures (11). In the case reported here the lesions of FCOD in patient's daughter develo-ped around the age of thirty years.

The sclerotic lesions are usually bilateral in maxilla and mandible, continuous throughout the tooth bearing areas of the jaws, with well defined radiolucent margins. Generally the lesions involve the alveolar bone posterior to the cuspids and this helps in differentiating from lesions of PCD which in-volve the periapex of the mandibular anterior teeth. Both the above lesions share similarities of age (middle age), sex (female), racial profile (blacks), comparable radiographic appearance (stages of ra-diolucent to radioopaque) and histologic appearance (2). The involved teeth in both the above lesions are vital.

Large FCOD lesions may displace the inferior alveolar canal in inferior direction, floor of the antrum in superior 
direction and result in buccal or lingual cortical expansion (3). In the case reported here the displacement of inferior alveolar canal as was more prominent in the left mandible and the floor of the antrum was obscured in the left maxilla (Fig. 2).

The internal structure may vary from radiolucent and radioopaque to complete radioopacity. The ra-dioopaque regions may vary from small well defined regions to large irregular regions (3) and some prominent radiolucent regions may be present which represent the development of simple bone cysts, which may enlarge in size or may fill with abnormal dysplastic cementoosseous tissue (1). Extensive lesions often have associated bony swelling and poorly localized intermittent pain in the affected bone (3). Moreover the single or multiple simple bone cysts may be interpreted as periapical pathology as in the case presented here of the patient's daughter and unnecessary endodontic treatment may be institu-ted with persistence of the periapical pathology after treatment (12). Attempted endodontic treatment in some cases with FCOD has resulted in flare up of the lesions at the periapex, therefore these cases have to be treated under antibiotic cover (13).

In the patient with symptoms the usual cause is infection, which produces chronic osteomyelitis; this may have features similar to chronic diffuse osteomyelitis (3), as seen in the case reported where in there was superimposed actinomycotic osteomyelitis in a long standing FCOD lesion. New symptoma-tic cases of FCOD may be difficult to distinguish from chronic diffuse osteomyelitis, but characteristic features of FCOD being asymptomatic with bilateral involvement of multiple quadrants of the jaw and confined to the alveolar bone may help in differentiating from (3) chronic diffuse osteomyelitis which is usually unilateral involving the mandibular body, angle and the ramus regions with widening of the mandibular canal, presence of sequestra as well as new periosteal bone formation (3).

The sclerotic areas of FCOD resembling "Cotton wool" is also seen in Paget's disease and chronic diffuse osteomyelitis radiographically. Paget's disease is polyostotic involving the jaws as well as the other bones such as the spine, femur, pelvis and sternum, and affects the entire bone of the mandible when compared to FCOD which is restricted to alveolar bone above the mandibular canal. Patient's with Paget's disease also present with elevated serum alkaline phosphatase levels (5). In the case reported here the serum alkaline phosphatase levels were well within normal limits and the sclerotic areas were restricted to the alveolar bone.

Multiple osteomas as in Gardner's syndrome has to be differentiated from sclerotic areas of FCOD, where in the former lesion involves bones other than the jaws and is also associated with multiple une-rupted supernumerary teeth in the jaws and multiple polyps of the small intestine $(3,14)$.

Management of symptomatic cases with drugs like clindamycin have been effective for anaerobic infections in bone, prior to which culture and antibiotic sensitivity tests (both aerobic and anaerobic) have to be done. Saucerization or partial surgical removal of large masses has not been beneficial, and reasonable efforts should be made to preserve teeth because a protracted clinical course of osteomyeli-tis and pain have followed elective extractions (3). Reconstruction of the surgical defect (vascularised grafts) has been reported to have $95 \%$ success. Implant supported prosthesis would improve the func-tional efficiency (4).

In the case of super imposed osteomyelitis HBO therapy has been proved to be beneficial which is administered as $100 \%$ oxygen at 2.5 atmospheric pressure for $120 \mathrm{~min}$ 5 days a week (15). HBO en-hances oxygen-dependent leukocyte killing through the production of hydrogen peroxide and super-oxide by providing increased oxygen tension in the hypoxic tissue. Secondly, optimal tissue oxygen tension enhances osteogenesis and neovascularization to fill the dead space with new bone and soft tissues (16). HBO has also been shown to enhance osteoaclastic activity to remove bony debris (16). Synergistic effects of HBO on bone healing with bone morphogenic protein were also demonstrated. Finally, HBO has also been shown to potentiate the antimicrobial effects of aminoglycosides, and possibly sulpha drugs and vancomycin, in the killing of susceptible bacteria (16).

Though HBO is the treatment of choice in chronic and recurrent osteomyelitis of mandible, it has not been used in cases of osteomyelitis associated with FCOD, which should be indicated in more number of cases in future.

\section{References}

1. Mupparapu M, Singer SR, Milles M, Rinaggio J. Simultaneous presentation of focal cemento-osseous dysplasia and simple bone cyst of the mandible masquerading as a multilocular radiolucency. Dentomaxillofac Radiol. 2005;34:39-43.

2. Mac Donald-Jankowski DS. Florid cemento-osseous dysplasia: a systematic review. Dentomaxillofac Radiol. 1996; 32:141-49.

3. Singer SR, Mupparapu M, Rinaggio J. Florid cement-osseous dysplasia and chronic diffuse osteomyelitis. Report of a simultaneous presentation and review of the literature. J Am Dent Assoc. 2005; 136 : 927-31.

4. Mangala M, Ramesh DN, Surekha PS, Santosh P. Florid cementoosseous dysplasia: Review and report of two cases. Indian J Dent Res. 2006; 17:131-4.

5. Gonçalves M, Píspico R, Alves Fde A, Lugão CE, Gonçalves A. Clinical, radiographic, biochemical and histological findings of florid cemento-osseous dysplasia and report of a case. Braz Dent J. 2005;16:247-50.

6. Melrose RJ, Abrams AM, Mills BG. Florid osseous dysplasia. A clinical-pathologic study of thirty-four cases. Oral Surg Oral Med Oral Pathol. 1976;41:62-82.

7. Jerjes W, Banu B, Swinson B, Hopper C. Florid cemento-osseous dysplasia in a young Indian woman. A case report. Br Dent J. 2005; 198: 477-8.

8. Harsha VB, Saraswathy K, Koteeswaran D. Florid cemento-osseous dysplasia. Indian J Dent Res. 2005;16:119-21.

9. Loh FC, Yeo JF. Florid osseous dysplasia in Orientals. Oral Surg 
Oral Med Oral Pathol. 1989;68:748-53.

10. Moshref M, Khojasteh A, Kazemi B, Roudsari MV, Varshowsaz $\mathrm{M}$, Eslami B. Autosomal dominant gigantiform cementoma associated with bone fractures. Am J Med Genet Part A. 2008; 146A:3246.

11. Rossbach HC, Letson D, Lacson A, Ruas E, Salazar P. Familial gigantiform cementoma with brittle bone disease, pathologic fractures, and osteosarcoma: a possible explanation of an ancient mystery. Pediatr Blood Cancer. 2005;44:390-6.

12. Islam MN, Cohen DM, Kanter KG, Stewart CM, Katz J, Bhattacharyya I. Florid cemento-osseous dysplasia mimicking multiple periapi-cal pathology--an endodontic dilemma. Gen Dent. 2008;56:55962.

13. Schneider LC, Mesa ML, Brickman JH. Complications of endodontic therapy in florid osseous dysplasia. Oral Surg Oral Med Oral Pathol. 1987 ; 64:114-6.

14. Lee BD, Lee W, Oh SH, Min SK, Kim EC. A case report of Gardner syndrome with hereditary widespread osteomatous jaw lesions. Oral Surg Oral Med Oral Pathol Oral Radiol Endod. 2009; 107:e68-72.

15. Chen CE, Shih ST, Fu TH, Wang JW, Wang CJ. Hyperbaric oxygen therapy in the treatment of chronic refractory osteomyelitis: a preliminary report. Chang Gung Med J. 2003: 26: 114-21.

16. Wang J, Li F, Calhoun JH, Mader JT. The role and effectiveness of adjunctive hyperbaric oxygen therapy in the management of musculoskeletal disorders. J Postgrad Med. 2002; 48: 226-31.

\section{Acknowledgement}

We acknowledge the contributions made by Dr. Veerendra Kumar B., Professor and Head, Depart-ment of Oral and Maxillofacial Pathology, Dr. Madura M.G. and Dr. Shaymala K. Lecturers, Depart-ment of Oral and Maxillofacial Pathology, DAPM R.V. Dental College and Hospital, Bangalore. 